\title{
Image Retrieval System in Heterogeneous Database
}

\author{
Khalifa Djemal, Hichem Maaref and Rostom Kachouri \\ University of Evry Val d'Essonne, IBISC Laboratory
}

France

\section{Introduction}

This chapter content try to give readers theoretical and practical methods developed to describes and recognize images in large database through different applications. Indeed, many computer vision system applications, such as computer-human interaction, controlling processes: autonomous vehicles, and industrial robots, have emerged a new need for searching and browsing visual information. Furthermore, due to the fast development of internet technologies, multimedia archives are growing rapidly, especially digital image libraries which represent increasingly an important volume of information. So, it is judicious to develop powerful browsing computer systems to handle, index, classify and recognize images in these large databases. Different steps can be composes an image retrieval system where the most important are, features extraction and classification.

Feature extraction and description of image content: Each feature is able to describe some image characteristics related to shape, color or texture, but it cannot cover the entire visual characteristics. Therefore, using multiple and different features to describe an image is approved. In this chapter, the extraction of several features and description for heterogeneous image database is presented and discussed. Test and choice of adequate classifiers to manage correctly clustering and image recognition: The choice of the used classifier in CBIR system (Content Based Image Retrieval) is very important. In this chapter we present the Support vector machines (SVMs) as a supervised classification method comprising mainly two stages: training and generalization. From these two important points, we try how we can decide that the extracted features are relevant in large heterogeneous database and the response to query image is acceptable. In these conditions we must find compromise between precision of image recognition and computation time which can be allowed to the CBIR System. In this aim, an heterogeneous image retrieval system effectiveness is studied through a comparison between statistical and hierarchical feature type models. The results are presented and discussed in relation with the used images database, the selected features and classification techniques. The different sections of this chapter recall and present the importance and the influence of the features relevance and classification in image recognition and retrieval system. Indeed, different features extraction methods (section 3) and classification approaches (section 4) are presented and 
discussed. We illustrate the principle and obtained results of the optimization methods in sections 5 .

\section{Related works}

The image recognition system, consists of extracting from a Database all the similar images to query images chosen by the user, figure 1 , has attracted much research interest in recent years. Principal difficulties consist on the capacity to extract from the image the visual characteristics, the robustness to the geometrical deformations and the quantification of the similarity concept between images. Indexation and recognition are given from a classification methods accomplished on images features. To understand the influence of the images database on the description method and the appropriate classification tool, it is more convenient to subdivide the image databases into two categories.

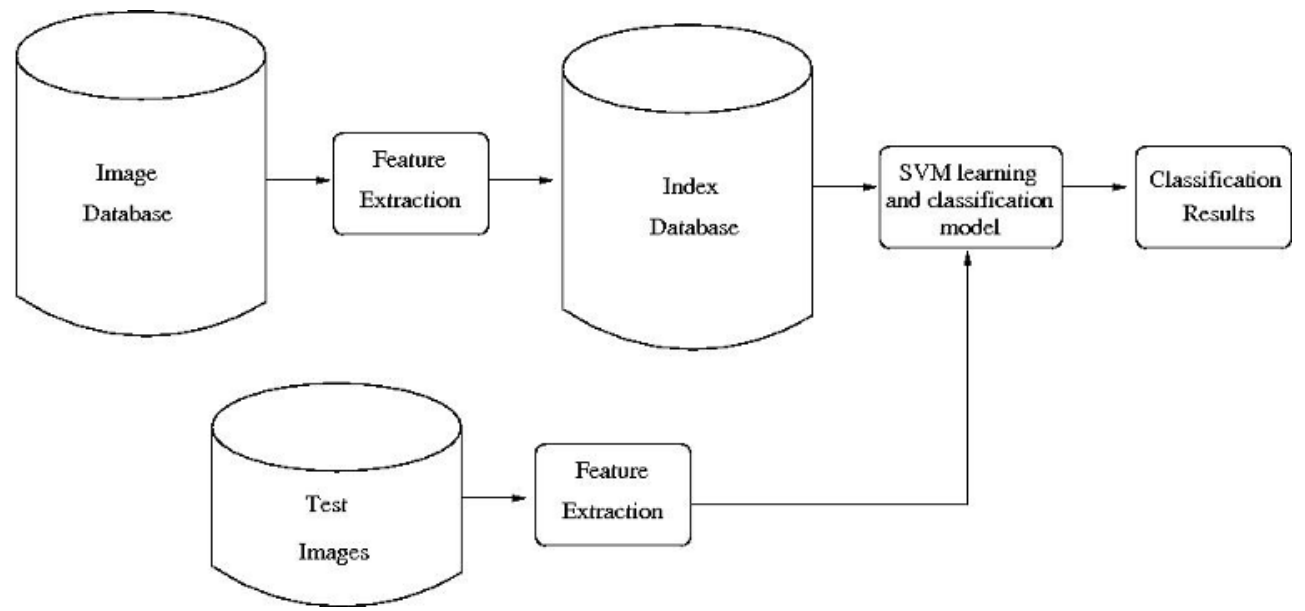

Fig. 1. Content Based Image Recognition system.

The first category consists of image databases usually heterogeneous. In this context, the objective of the images recognition system is to assist the user to intelligently search in images database a particular subject adapting to the subjective needs of each user. The second category concerns specific image databases. In this context, the images are most often a uniform semantic content. The concerned applications are generally professional. To index this image databases, the user must integrate more information defined by the expert to develop a specific algorithm, the objective is to optimize system efficiency and its ability to respond as well as the expert. This categorization is to be taken into account when developing any content image retrieval system. Indeed, to obtain satisfactory results, the choice or development of new description methods must be appropriate for the type of the considered images database. This is due simply to the great difficulty to obtain a universal description algorithm.

The content-based image retrieval that we have just described it in figure 1, have attracted the attention of several specialists in different domains and caused for a few years an important effort of research. Indeed, there has been growing interest in indexing large 
biomedical images by content due to the advances in digital imaging and the increase development of computer vision. Image recognition in biomedical databases, for example, are critical assets for medical diagnosis. To facilitate automatic indexing and recognition of image databases, several methods has been developed and proposed. As described above, two important steps composed an image retrieval system, features extraction and classification. We present in this section some important related work to these stages. We find several choices of features from the low level to high level: shape, geometry, symbolic features, .etc. The basic goal in content-based image retrieval and recognition is to bridge the gap from the low-level image properties. Consequently, we can directly access to the objects, and users generally want to find in image databases. For example, color histograms (Stricker \& Swain, 1994), (Swain \& Ballard, 1991), are commonly used in image description and have proven useful, however, this global characterization lacks information about how the color is distributed spatially. Several researchers have attempted to overcome this limitation by incorporating spatial information in the features extraction stage. Stricker and Dimai (Stricker \& Dimai, 1997), store the average color and the color covariance matrix within each of five fuzzy image regions.

(Huang et al., 1997) store a color correlogram that encodes the spatial correlation of colorbin pairs. (Smith \& Chang, 1996), store the location of each color that is present in a sufficient amount in regions computed using histogram back-projection. (Lipson et al., 1997) retrieve images based on spatial and photometric relationships within and across simple image regions. Little or no segmentation is done; the regions are derived from lowresolution images. In (Jacobs et al., 1995), authors use multiresolution wavelet decompositions to perform queries based on iconic matching. Some of these systems encode information about the spatial distribution of color features, and some perform simple automatic or manually-assisted segmentation. However, none provides the level of automatic segmentation and user control necessary to support object queries in a very large image databases. (Carson et al., 2002), see image retrieval ultimately as an object recognition problem and they proceed in three steps. Firstly, pixels into regions which generally correspond to objects or parts of objects were grouped. These regions are described in ways that are meaningful to the user. The proposed system allows access to these region descriptions, either automatically or with user intervention, to retrieve desired images. In this approach the features do not encode all the important information in images and the image retrieval is obtained without classification what can poses a problem of recognition in large image databaes. (Antani et al., 2002), presents a comprehensive survey on the use of pattern recognition methods which enable image and video retrieval by content where the classification methods are considered. Research efforts have led to the development of methods that provide access to image and video data. These methods have their roots in pattern recognition. The methods are used to determine the similarity in the visual information content extracted from different low level features.

A multi-level semantic modeling method is proposed in (Lin et al., 2006), which integrates Support Vector Machines (SVM) into hybrid Bayesian networks (HBN). SVM discretizes the continuous variables of medical image features by classifying them into finite states as middle-level semantics. Based on the HBN, the semantic model for medical image semantic retrieval can be designed at multi-level semantics. To validate their method, a model is built to achieve automatic image annotation at the content level from a small set of astrocytona MRI (magnetic resonance imaging) samples. Indeed, Multi-level annotation is a promising 
solution to enable image retrieval at different semantic levels. Experiment results show that this approach is very effective to enable multi-level interpretation of astrocytona MRI scan. This study provides a novel way to bridge the gap between the high-level semantics and the low-level image features.
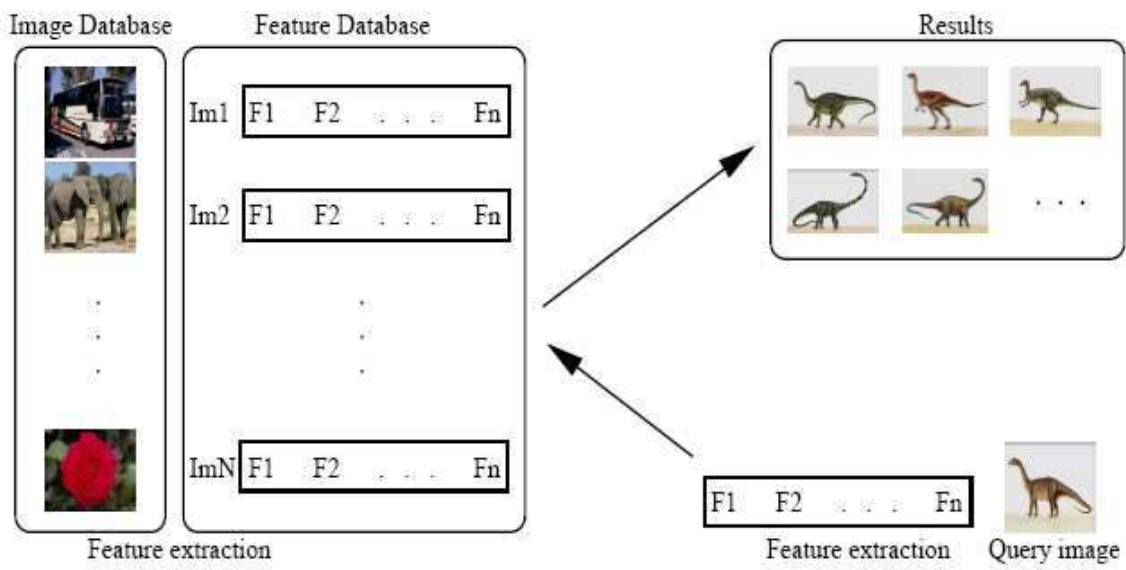

Fig. 2. Features extraction and image recognition: form a query image, a relevant features extraction allows to obtain all similar images in database.

Many indexation and recognition systems were developed based on image content description and classification in order to perform image recognition in large databases (figure 2). These systems use low level features such as the colors and orientations histograms, Fourier and wavelet transforms. In spite of these acceptable results, the classification based on a similarity distance computing is not enough robust to manage great dimensions of the extracted features vectors. To resolve this problem, other proposed systems calculate for each pixel of each image a characteristics vector. This vector contains the components associated with the color, the texture and position descriptors, which gives a better description of the image. But the performances of the image description remain to be improved. Moreover, several works were based on the wavelet tools. The authors in (Serrano et al., 2004), have enhanced their image representation by the use of the texture features extracted by wavelet transform. A new extraction technique of rotation invariants is proposed in (Sastry et al. 2004), this method offers satisfactory results, taking into account the rotation features. For more precision and representativeness of images, a new transform called Trace transform is proposed in (Kadyrov \& Petrou, 2001). This transform offers at the same time a good description of image and is invariant to rotation, translation and scaling. After the features extraction, classification is made afterwards by the means of a classifier, such as KNN classifier. But this classifier is slow considering his incapacity to manage great dimensions of feature vectors. A more effective method based on a Bayesian approach (Sclaroff \& Pentland, 1995), which consists in concatenation of feature blocks gave better results. The artificial training techniques were also used in the field of the image classification (artificial neural networks (ANN), genetic algorithms,. . . etc). Their broad use is due to the facilities which they offer to the level computing time and their performances 
in term of classification. Indeed, we find many works based on the ANN. The obtained results show a great capacity thanks to the speed offered by the ANN and the simplicity of their implementation (Takashi \& Masafumi, 2000), (Egmont-Petersen et al. 2002). In (Djouak et al., 2007), a combination of features vectors is proposed. These vectors are obtained by a visual search based on the colors histograms, the geometrical and texture features. The rotation invariants and texture features are given using wavelet transform. In addition, the Trace transform is introduced in order to obtain more invariance. A good compromise between effectiveness and computation simplicity are obtained using RBF classification technique. While every content heterogeneous image recognition system, as mentioned above, has two stages: Extraction of discriminate features and classification (figure 1), feature extraction is the most important one, because satisfactory image classification rely basically on a better image description.

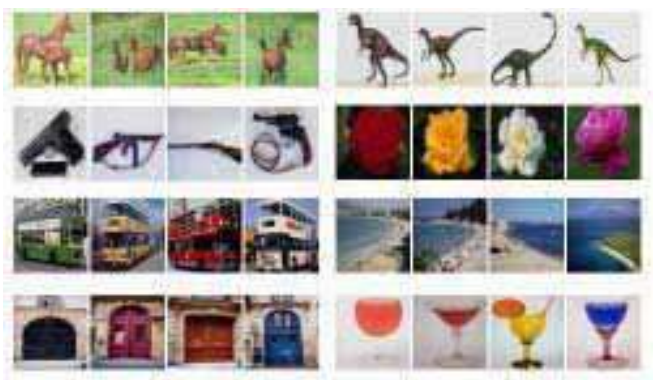

Fig. 3. Images database sample.

In fact, classification algorithm choice is generally based on index content data-sets. Moreover in case of heterogeneous image databases, relevant feature selection is recommended. In section 3, we discuss feature extraction and selection. We evaluated our modular statistical optimization and hierarchical type features model, presented in section 5 on a very well-known heterogeneous images database, chosen from the Wang image database available on this Web site: http://wang.ist.psu.edu/docs/home.shtml/ This image collection contains 1000 images of various types having large difference in colors, shapes, and textures. Some samples are shown in figure 3.

In this chapter, we present in section 3 and 4 the two important steps of an image recognition and retrieval system. In section 5, two CBIR systems are proposed, the first one is based on modular statistical methods and the second on hierarchical best features type selection.

\section{Features extraction}

In this section, several feature extraction and evaluation for an heterogeneous image database recognition are detailed. Indeed, the main objective of feature extraction is to find, for each image, a representation (signature) that is, in one hand compact to be quickly accessible and easily comparable, and in the other hand enough comprehensive to well characterize the image. Most used features, mainly, reflect the low level characteristics in image, such as color, texture, and/or shape (Bimbo 2001). Color features are the first used in 
CBIR systems, and they still be the most used due to their extraction simplicity, rich description and their recognition efficiency. Average color (Faloutsos et al. 1994) and color histograms (Hafner et al. 1995) are very useful, they are position and scale variation insensitive. Correlogram feature was proposed by (Huang et al. 1997) as improvement of color histogram. It is a matrix that describes the spatial correlation between colors in an image according to some distance and a certain degree of orientation. Then, the autocorrelogram, a sub-feature of the correlogram one was defined in (Huang et al. 1999), it captures only the spatial auto-correlation between the same color intensities in the image. (Bimbo 2001), have provided an extensive study of different color indexing methods. Also, a set of color features was tested for inclusion in the standard MPEG-7 (Manjunath et al., 2001). Texture is increasingly used in image indexing, because it mitigates certain problems arising from color indexing, in particular when the color distributions are very close. The existing texture descriptors can be classified into two categories: The first one is deterministic and refers to a spatial repetition of a basic pattern in different directions. This structural approach corresponds to macroscopic textures. First order statistics (Press 1987) is one of the most simple feature computations of this kind. It is extracted from the normalized image histogram. Co-occurrences matrix proposed by (Haralick \& Dinstein, 1973) is also used to analyze texture in the spacial field. This matrix allows to compute the same gray level pixel numbers in the image, which are separated by certain distance and positioned according to certain direction. From this matrix, thirteen texture features are computed (Haralick \& Dinstein 1973). The second category of texture is probabilistic, it seeks to characterize the chaotic aspect which does not include localizable pattern or main repetition frequency. This approach is called microscopic, or multi-resolution. Examples of texture features derived from the wavelet transformation are presented in (Serrano et al. 2004). As color feature, a set of texture features was tested for inclusion too in the standard MPEG-7 (Manjunath et al. 2001). In addition of color and texture feature, which describes the general aspect of an image, shape features are able to characterize the different objects in the image. Generally, this kind of feature indicates the object outline, then segmentation as image preprocessing is often necessary, moreover reliable segmentation is especially critical for shape feature characterization. Image segmentation subdivides image into regions of interest allowing its understanding or further processing. Ones region segmentation is realized, two shape feature kinds could be extracted (Chen et al. 2004), (Chen et al. 2006). The first is based on image region geometry. The second is based on pixel grey level statistics in different image regions. Among the most popular features of this kind, we cite invariant moments of $\mathrm{HU}(\mathrm{Hu}$ 1962). These moments are invariant to different geometric transformation (translation, rotation and scale variation). In our case, because of the complex heterogeneous image contents, a rich description covering the different visual characteristics is needed (Manjunath et al. 2001). For this, we employ in this chapter a set of features belonging the three visual kinds: We use the four average color feature vectors (Faloutsos et al. 1994) computed in different color spaces, histograms, and correlogram (Huang et al. 1997), (Huang et al. 1999), as color features. First order statistics (Press 1987), cooccurence matrix (Haralick \& Dinstein 1973) and Daubeshies wavelet transformation are used as texture features. The gradient norm (Delingette \& Montagnat 2001) and the invariant moments of $\mathrm{Hu}(\mathrm{Hu}$ 1962) are used as shape features. Figures 4, 5 and 6 show some extracted feature samples. 


\subsection{Used and improved features}

We present in this section the different used and improved features. Three types of features are discussed, color, textures and shape features. The improvement is given from gradient norm projection and $\mathrm{Hu}$ moments considered and obtained from gradient norm image after the suppression of the local maxima.

\subsubsection{Color features}

Fist we compute the average color in the RGB, HSV, HMMD and $\mathrm{YCbCr}$ spaces.

$$
R_{\text {avg }}=\frac{1}{N} \sum_{p=1}^{N} R(p), \quad G_{a v g}=\frac{1}{N} \sum_{p=1}^{N} G(p), \quad B_{a v g}=\frac{1}{N} \sum_{p=1}^{N} B(p)
$$

Where $X=\left(R_{\text {avg }}, G_{\text {avg }}, B_{\text {avg }}\right)^{T}$ and $N$ the image pixel number. The second color features used are the histograms:

$$
\begin{gathered}
\operatorname{histR}(x)=\frac{1}{N} \sum_{p=1}^{N} R\left(x_{p}=x\right) \quad \operatorname{hist} G(x)=\frac{1}{N} \sum_{p=1}^{N} G\left(x_{p}=x\right) \\
\operatorname{histB}(x)=\frac{1}{N} \sum_{p=1}^{N} B\left(x_{p}=x\right)
\end{gathered}
$$

With $x \in[0,1,2, \ldots \ldots ., 255]$.

We use the color autocorrelogram, defined in (Huang et al. 1999), captures the spatial correlation between identical colors only, it is a subset of the correlogram (Huang et al. 1997) which expresses how the spatial correlation of pairs of colors changes with distance.

\subsubsection{Textures features}

As texture features we use the First Order Statistics, the Daubeshies Wavelett transform and the Spatial Gray Level dependence matrix.

The first order statistics are considered by the mean, the standard deviation and the coefficient of variation.

$$
\begin{gathered}
\text { Mean }=\frac{1}{255} \sum_{x=0}^{255} x \cdot h i s t(x), \quad S \text { tandardDeviation }=\frac{1}{255} \sum_{x=0}^{255} x-\text { Mean }^{2} \cdot h i s t(x) \\
\text { CoefficientOfVariation }=\frac{S \tan \text { dardDeviation }}{\text { Mean }}
\end{gathered}
$$

The Daubeshies Wavelett transform in the fourier space is given by the following equations:

$$
\hat{\varphi}(\omega)=\Pi_{k=1}^{\infty} \frac{h\left(2^{-k} \omega\right)}{\sqrt{2}}
$$


$\hat{\psi}(\omega)=\frac{1}{\sqrt{2}} g\left(\frac{\omega}{2}\right) \hat{\varphi}\left(\frac{\omega}{2}\right) \quad h(\omega)=\sum_{k \in Z} h_{k} e^{-i k \omega} \quad g(\omega)=\sum_{k \in Z} g_{k} e^{-i k \omega}$

And the decomposition is given as follow:
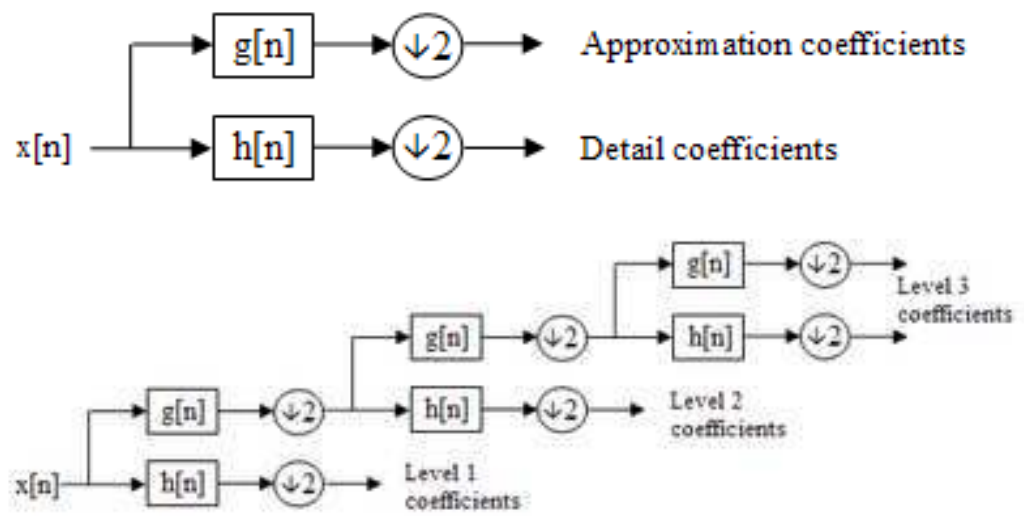

The Wavelet coefficients are $c_{i j}(x, y)$, where $l$ is the decomposition level.

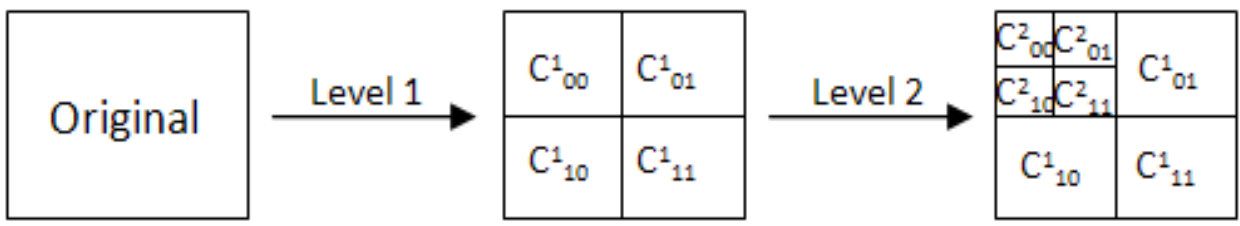

The lowest frequency coefficients $c^{2} 00(x, y)$ are not inherently useful for texture analysis. Therefore, a direction-independent measure of the high-frequency signal information is obtained by filtering the raw coefficients $c^{2}{ }_{00}(x, y)$ with the Laplacian. The texture features are obtained by computing the subband energy of all wavelet coefficients (including the Laplacian filtered $c^{2} 00(x, y)$ coefficients) :

$$
e_{i j}^{l}=\frac{1}{M N} \sum_{m=1}^{M} \sum_{n=1}^{N}\left|C_{i j}^{l}(m n)\right|^{2}
$$

where $\mathrm{M}$ and $\mathrm{N}$ are the dimensions of coefficients $c_{i j}(x, y),(\mathrm{Wu}, 2003)$.

For the Spatial Gray Level dependence matrix (SGLD Matrix), the co-occurrence matrix (Julezs, 1975), called also the spatial gray level dependence (SGLD) matrices, counts how often pairs of gray level of pixels, separated by a certain distance and lying along certain direction, occur in an image. From these SGLD matrices, thirteen features related to the image texture, could be calculated, (Haralick \& Destein, 1973). 


\subsubsection{Shape features}

The invariant moments of $\mathrm{Hu}$ and the Gradient norm projection are used as shape features. The $\mathrm{Hu}$ invariants $(\mathrm{Hu}, 1962)$ are obtained from quotients or powers of moments. A moment is a sum over all pixels of the image model by weighted polynomials related to the positions of pixels. Let $I(x, y)$ the grayscale of a pixel in the image $I$, we define the moment of order $(p+q)(p, q>0)$ of an image I by:

$$
m_{p, q}=\int_{R^{2}} x^{p} y^{q} I(x, y) d x d y
$$

Let $\left(x_{0,}, y_{0}\right)$ the centroid of the function $I$ and the centered image $I_{T}(x, y)=I\left(x+x_{0}, y+y_{0}\right)$ is invariant by translation. The central moment of order $(p+q)$ of the function $I$ is written as follows:

$$
v_{p, q}=\int_{R^{2}} x^{p} y^{q} I\left(x+x_{0}, y+y_{0}\right) d x d y
$$

The central moments are invariant by translation. We introduce the normalized moments as follows:

$$
\mu_{p, q}=\frac{v_{p, q}}{v_{0,0}^{(1+(p+q) / 2)}}
$$

These moments are invariant to translation and scale changes. Hu moments are calculated from normalized moments and are invariant by translation, rotation and change of scale:

$$
\begin{aligned}
& \phi_{1}=\mu_{2,0}+\mu_{0,2} \\
& \phi_{2}=\left(\mu_{2,0}-\mu_{0,2}\right)^{2}+4 \mu_{1,1}^{2} \\
& \phi_{3}=\left(\mu_{3,0}-3 \mu_{1,2}^{2}+\left(3 \mu_{2,1}-\mu_{0,3}\right)^{2}\right. \\
& \phi_{4}=\left(\mu_{3,0}+\mu_{1,2}\right)^{2}+\left(\mu_{2,1}+\mu_{0,3}\right)^{2} \\
& \phi_{5}=\left(\mu_{3,0}-3 \mu_{1,2}\right)\left(\mu_{3,0}+\mu_{1,2}\right)\left[\left(\mu_{3,0}+\mu_{1,2}\right)^{2}-3\left(\mu_{2,1}+\mu_{0,3}\right)^{2}\right] \\
& +\left(3 \mu_{2,1}-\mu_{0,3}\right)\left(\mu_{2,1}+\mu_{0,3}\right)\left[3\left(\mu_{3,0}+\mu_{1,2}\right)^{2}-\left(\mu_{2,1}+\mu_{0,3}\right)^{2}\right] \\
& \phi_{6}=\left(\mu_{2,0}-\mu_{0,2}\right)\left[\left(\mu_{3,0}+\mu_{1,2}\right)^{2}-\left(\mu_{2,1}+\mu_{0,3}\right)^{2}\right]+4 \mu_{1,1}\left(\mu_{3,0}+\mu_{1,2}\right)\left(\mu_{2,1}+\mu_{0,3}\right) \\
& \phi_{7}=\left(3 \mu_{2,1}-\mu_{0,3}\right)\left(\mu_{3,0}+\mu_{1,2}\right)\left[\left(\mu_{3,0}+\mu_{1,2}\right)^{2}-3\left(\mu_{2,1}+\mu_{0,3}\right)^{2}\right] \\
& -\left(\mu_{3,0}-3 \mu_{1,2}\right)\left(\mu_{2,1}+\mu_{0,3}\right)\left[3\left(\mu_{3,0}+\mu_{1,2}\right)^{2}-\left(\mu_{2,1}+\mu_{0,3}\right)^{2}\right]
\end{aligned}
$$

The Gradient Norm projection feature is obtained after some processing steps. First, we apply Sobel masks to obtain the directional gradients according to $\mathrm{x}$ and $\mathrm{y}$ :

$$
G_{x}(i, j)=h_{x}(i, j) \otimes I(i, j) \quad G_{y}(i, j)=h_{y}(i, j) \otimes I(i, j)
$$

where $I(i, j)$ is the image gray level information and $h_{x}(i, j), h_{y}(i, j)$ are Sobel masks: 


$$
h_{x}(i, j)=\left[\begin{array}{ccc}
-1 & -2 & -1 \\
0 & 0 & 0 \\
1 & 2 & 1
\end{array}\right] \quad h_{y}(i, j)=\left[\begin{array}{ccc}
-1 & 0 & -1 \\
-2 & 0 & -2 \\
-1 & 0 & -1
\end{array}\right]
$$

then, gradient norm is computed as follow:

$$
G(i, j)=\sqrt{G_{x}(i, j)^{2}+G_{y}(i, j)^{2}}
$$

As the coefficient number in the gradient norm is the same as the pixel number in the image, we compute the gradient norm projection according to $\mathrm{x}$ and $\mathrm{y}$, in order to reduce this feature size:

$$
P_{X i}=\frac{1}{\max G_{i, j}} \sum_{j} G(i, j) \quad P_{Y i}=\frac{1}{\max G_{i, j}} \sum_{j} G(i, j)
$$

\subsection{Examples of description results and discussion}

From the presented variety feature vector extraction, an improvement of certain vectors (Kachouri et al., 2008b) is ensured, in order to reduce their size while reserving their relevance description and even improve it.
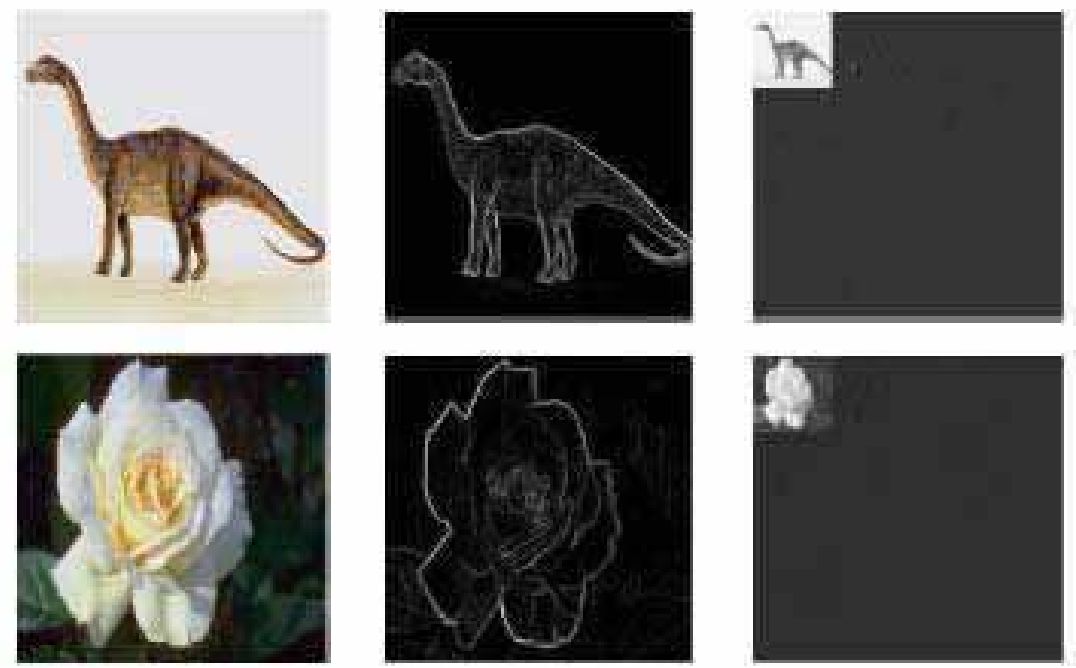

a)

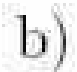

C)

Fig. 4. Extracted feature samples: a) Dinosaur and Flower images , b) Dinosaur and Flower image respectively gradient norm and c) Dinosaur and Flower image respectively two level decomposition Daubechies wavelet coefficients.

For this, we employ the auto-correlogram, computed from correlogram feature (Huang et al. 1999), we retain only the eight most commonly used features since thirteen extracted from 
image co-occurrence matrix. Also a condensation to compute single value resulting from different level decomposition detail coefficients of the Daubeshies wavelet transformation and the Laplacian approximation filtering, is made, figure 4.
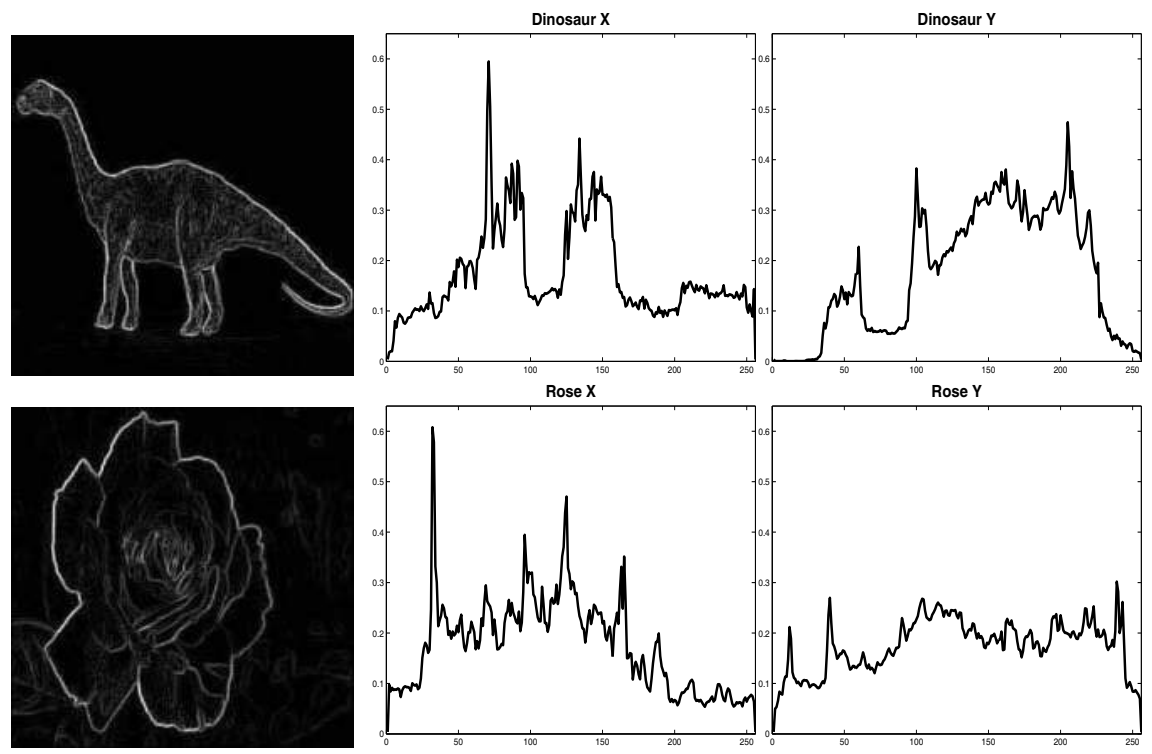

Fig. 5. Extracted features samples: Gradient Norm projection (/x and /y), obtained from Dinosaur and Rose images.

Moreover we use instead of the initial gradient norm matrix, just a gradient norm projection according to $\mathrm{x}$ and $\mathrm{y}$ axes, which allow to reduce the dimension of this feature while reserving its same description quality. Figure 5 shows discrimination capability of this new feature between images from different classes.

Finally, we retain, from seven extracted, only the first four invariant $\mathrm{Hu}$ moments. In addition, these moments are computed from the gradient norm instead of the initial pixel values. This helps to consider the contour more than other information in the image which enhances furthermore the shape description of this feature (figure 6).

This information features will help to build models of objects for the recognition and indexing in a database. To properly accomplish this task, we need a classification system able to categorize all objects and images in a database from their description. In the rest of this chapter, we note the different average color vectors as RGB, HSV, HMMD and $\mathrm{YCrCb}$, histograms as Hist, auto-correlogram as Auto-Corr, first order statics as FOS, co-occurence matrix as SGLD, Daubeshies wavelet transformation as Daub, gradient norm as G-norm and the invariant moments of $\mathrm{Hu}$ as $\mathrm{Hu}$. 

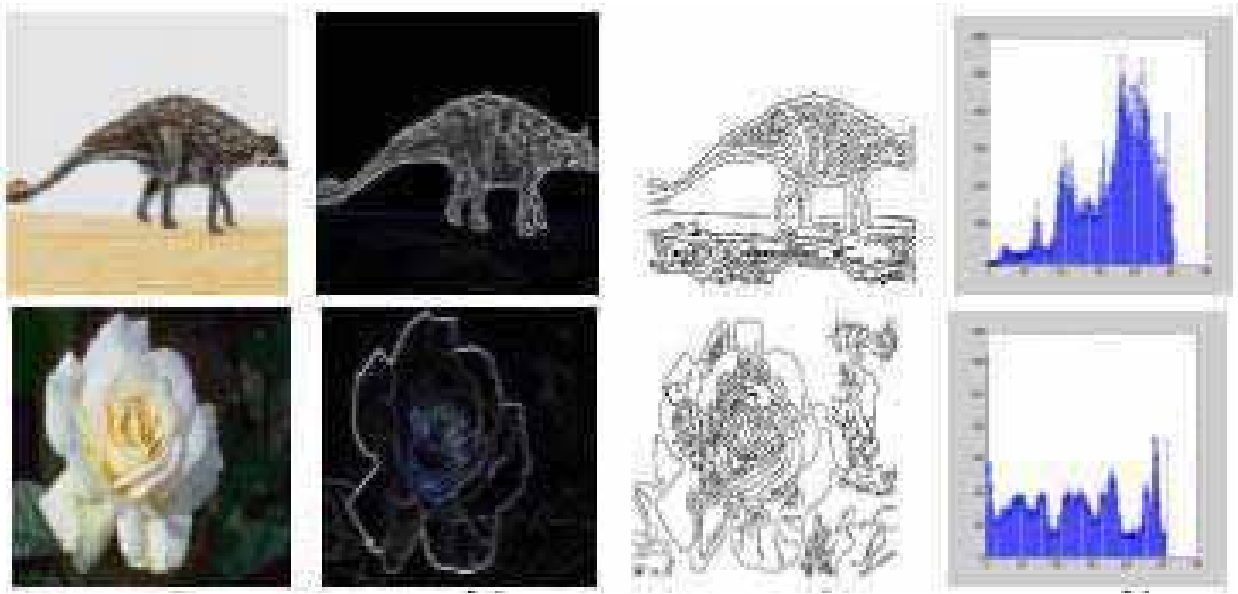

Fig. 6. Contour extraction and Histograms examples: From real images, we compute first the gradient norm, the contours extraction, the histograms and the Hu moments.

\section{Classification}

We call automatic classification, the algorithmic categorization of the objects and images. This one is to determine in which class or category you can "store" each object, based on similarities criteria. Many classification or learning methods have been already developed, and used in a wide spectrum of applications: diagnosis, bioinformatics, fraud detection, speech recognition, etc.

Once training image feature set is built, a classifier is used to ensure image recognition. There are two classification kinds in the literature. The first is unsupervised classification where the classifier deals directly feature dataset without any prior knowledge (Kachouri et al. 2008c). Classification here is based, generally, on clustering approach. So, CBIR systems allow image access according to their visual characteristics by means of similarity measures. The smaller the similarity distance is, the closer the two images are. The most used distance in literature is the Euclidian one which is really a particular case of the Minkowski metric (Kachouri et al. 2008c), various similarity measures are discussed in (Ramesh et al. 1995). The second type is supervised classification (Kachouri et al. 2008a), where the classifier assumes that there is already image classification, a training stage is required, it is the case of a library or a search engine. Since the Nineties, Support vector machines (SVMs) did not cease arousing the interest of several researcher communities of various expertise fields. Nowadays, SVM performance, in several applications (Bi et al. 2003), (Chen et al. 2006), exceeds those of already established traditional models. In fact, SVM originally formulated for twoclass classification problems, have been successfully extended for multiclass classification problems, and become in a very short period of time the standard state of-theart tool. Also in the SVM-multiclass, original input space is mapped into a higher dimensional feature space in which an optimal separating hyper-plane is constructed on the basis of Structural Risk Minimization (SRM) to maximize the margin between the different clusters, i.e., generalization ability. 


\subsection{Support Vector Machines (SVM)}

This method of supervised classification was published by (Vapnik, 1998). An SVM is a supervised learning algorithm allows to learning a separator. We have a finite set of separated vectors of $R^{n}$, as part of a binary classification into two groups, or two classes. Learning to a group is defined by an associated label to each vector, which is inscribed group 1 or group 2 . Find a separator is equivalent to reconstruct a function that takes a vector from a set of samples and can say what group it belongs. However, it expects the SVM good generalization properties, ie if a new vector is introduced and it was not in the set of database, the SVM will say which group it is probably belongs.

The linear separator is the lower part of the SVM, that is a relatively simple problem, and that allows later on to the SVM providing some well more powerful separators. We saw that we started from a finite set of labelled vectors. We say that all the labelled vectors that we give are the set of samples noted $S$, which contains $p$ elements.

$$
S=\left(\vec{x}_{l}, y_{l}\right)_{1 \leq l \leq p} \text { with } \forall l, y_{l} \in\{-1,1\}
$$

The scalar product of two vectors is noted where is $\langle\vec{x}, \vec{y}\rangle$, we can then define the linear separator $f_{\vec{w}, b}$ by the following equation:

$$
f_{\vec{w}, b}(\vec{x})=\langle\vec{w}, \vec{x}\rangle+b
$$

This separator does not provide valid values only -1 or 1 , but we assume that the result when $f_{\vec{w}, b}(\vec{x})$ is positive, then the vector is of the same class as samples of label 1 , and the result when $f_{\vec{w}, b}(\vec{x})$ is negative, the vector belongs to the class of examples labelled -1 . The equation $f_{\vec{w}, b}(\vec{x})=0$ defines the border between the two classes. This boundary is an affine hyper-plane in the case of linear separator.

\subsubsection{Separability and margin}

We suppose that $S$ is linearly separable. The basic idea of SVM is to separate samples of each class, but it is also necessary that the hyper plane passes as possible between the two classes. It is to define this notion that we introduce the margin. The margin is the minimum distances of each sample to the hyper plane (figure 7).
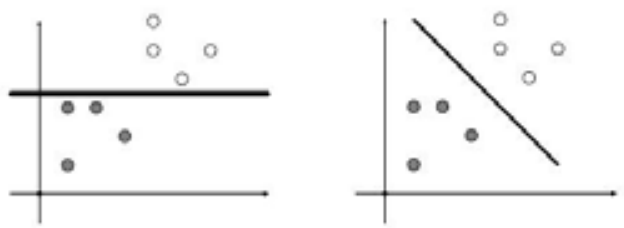

Fig. 7. A separator that the margin is not maximized, have less good generalization properties than that which the margin is maximized. 
The aim of the SVM, in case where $S$ is separable, is to give separator $S$ whose margin is maximal, while ensuring that properly separates the samples with label -1 and the samples with label 1.

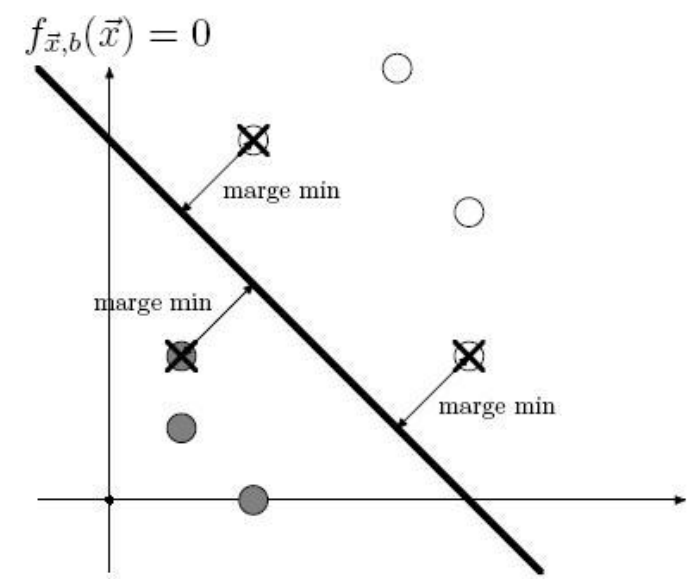

Fig. 8. The separator which should maximize the margin.

The maximum margin separator is such that, the smaller sample has a margin wider than the sample of the smallest margin of the other possible separators. In fact, there are really at least two samples of smaller margin, a class 1 and class -1 . They force this margin, and the border of separation passes between them (figure 8). These are the only samples that force the margin, and remove all other samples of the database does not change the separator. These samples are called support vectors, hence the name of the method.

\subsubsection{General case}

For the general case where $S$ is not separable, the solution is to allow some samples to have a lower margin than the margin chosen as the smallest margin or even negative. However, the solution of the problem may be very bad if too many samples are allowed to have a small margin. The idea is to add value margins lower than the maximum margin in the expression to minimize. This avoids that the margins are too low, which limits the samples that do not respect the separability through a separator solution of optimization problem. This is a problem of quadratic convex optimization, i.e. an optimization problem that admits no local optimum, but only one optimum, thus overall. This is crucial because the convexity of the problem is a guarantee of convergence to the SVM solution.

The interest of the kernel functions is that they allow using what we just presented on the linear separation to the non-linear separations. Let $S$ a set of samples labelled by 1 or -1 depending on the class to which they belong, which is not at all linearly separable. The method we have seen works in this case but may give poor results, and many samples became support vectors. The idea of using kernels comes from the assumption that if a set is not linearly separable in the descriptors space, it can be in a space of higher dimension. A better way to separate the samples is to project them into a different space, and perform a 
linear separation in this space, where this time it should be more adapted. The kernel functions can achieve this projection, and must check a number of properties to ensure the effectiveness of this technique, so you do not have to make calculations in very large dimensions. With the kernel functions, we can work in very large dimensions. However, a linear separation, and a linear regression is facilitated by the projection of data in a space of high dimension. Projecting in the space of descriptors and using an algorithm to maximize the margin, SVM managed to get a severability retaining good generalization capacity, is the central idea of SVM.

For more details on SVMs, we refer interested readers to (Cristianini \& Taylor, 2000). A comparison between SVM-multiclass, as supervised classification and Euclidian distance based k-means, as unsupervised classification, is presented in (Kachouri et al., 2008b). The obtained results prove that SVM classifier outperforms the use of similarity measures, chiefly to classify heterogeneous image database. Therefore, we integrate SVM classifier in our proposed image retrieval systems in this chapter.

\section{Image recognition and retrieval results through relevant features selection}

To ensure a good feature selection during image retrieval, we present and discuss the effectiveness of the different feature kind and aggregation. Since heterogeneous image database contains various images, presenting big content difference. The idea to introduce a system optimization tool was essential when one realized during the carried out tests that the use of all extracted features could be heavy to manage. Indeed, more features vectors dimensions are significant more the classifier has difficulties for their classification. The traditional way that one followed in (Djouak et al., 2005a) and that one finds in many CBIR systems is a diagram which consists of the use of all extracted features in the classification step. Unfortunately, this method presents a great disadvantage, by using all features the classifier manages a great dimensions number. That involves a consequent computing time what creates a real handicap for great images databases. In fact, this problem which is the direct result of the high dimensionality problem was the subject of several works which led to cure it.

Feature (content) extraction is the basis of CBIR. Recent CBIR systems retrieve images based on visual properties. As we use an heterogeneous image database, images are various categories, and we can find a big difference between their visual properties. So a unique feature or a unique feature kind, cannot be relevant to describe the whole image database.

Moreover, while SVM is a powerful classifier, in case of heterogeneous images, given the complexity of their content, some limitations arise, it is that many features may be redundant or irrelevant because some of them might not be responsible for the observed image classification or might be similar to each other. In addition when there are too many irrelevant features in the index dataset, the generalization performance tends to suffer. Consequently, it becomes essential and indispensable to select a feature subset that is most relevant to the interest classification problem. Hence the birth of a new issue, other than image description, it is relevant feature selection. Subsequently, to guarantee a best classification performance, good content image recognition system must be, mainly, able to determine the most relevant feature set, then to well discretize correspond spaces. Feature selection for classification purposes is a well-studied topic (Blum \& Langley 1997), with some recent work related specifically to feature selection for SVMs. Proposed algorithms in 
this regard, shall be ample literature for several years (Guyon \& Elisseeff 2003). Although proposed selection methods, are quite varied, two main branches are distinguished, wrappers and filters (John et al. 1994), (Yu \& Liu 2004). Filtres are very fast, they rely on theoretical considerations, which allow, generally, to better understanding variable relationships. Linear filters, as PCA (Principal Component Analysis), or FLD (Fisher's Linear Discriminant) (Meng et al. 2002) are very used, but these methods are satisfactory, only if there is a starting data redundancy. (Daphne \& Mehran 1996) propose markov blanket algorithms, which allow to found for a given variable $x i$, a set of variables not including $x i$ that render xi un-necessary. Once a Markov blanket is found, xi can safely be eliminated. But this is only a summary approximation, because this idea is not implementable in practice. However, as it does not take into account the used classifier in generalization stage, all filters kind selection methods still, generally, unable to guarantee high recognition rate. Although conceptually simpler than filters, wrappers are recently introduced by (John et al. 1994). This selection kind uses the classifier as an integral part of the selection process. Indeed, the principle of a feature subset selection is based on its success to classify test images. Therefore, the selected feature subset is well suited to the classification algorithm, in other words, high recognition rates are obtained because selection takes into account the intrinsic bias of classification algorithm. Some specifically related works on feature selection using SVM classifier are recorded in literature (Guyon et al. 2002), (Zhu \& Hastie 2003), (Bi et al. 2003), (Chen et al. 2006). The major inconvenient of this selection technique is the need for expensive computation, especially when the variable number grows. More details, are accommodated in (Guyon \& Elisseeff 2003) and references therein. To take advantage, of both of these selection kinds, filters speed and selected feature subset adaptability with the used classifier in wrappers, new selection methods ensuring that compromise is always looking. Recently, (Bi et al. 2003) have proposed the use of 1-norm SVM, as a linear classifier for feature selection, so computational cost will not be an issue, then non linear SVM is used for generalization. Other methods combining filters and wrappers are presented in (Guyon \& Elisseeff 2003). It is within this framework that we propose in this section, the modular statistical optimization (section 5.1) and the best features type selection (section 5.2) methods.

\subsection{Modular statistical optimization}

The proposed modular statistical architecture in figure 9 is based on a feedback loop procedure. The principal idea (Djouak et al., 2006) of this architecture is that instead of using all features in the classification step, one categorizes them on several blocks or modules and after one tries to obtain the optimal precision with the minimum of blocks. The introduced modular features database includes all presented features in section 3 .

Using all these features one formed four features modules which one can describe as follows: The first module (b1) gathers the all shape features, the second module (b2) gathers the color features, the third module (b3) the texture features and finally the fourth module (b4) the Daubeshies features.

\begin{tabular}{|l|l|l|l|l|l|c|}
\hline Features Blocs & B1 & B2 & B3 & B4 & B5 & B6 \\
\hline Concerned Modules & b1 & b1 b2 & b3 & b1 b2 b3 & b4 & b1 b2 b3 b4 \\
\hline
\end{tabular}

Table. 1. Used features blocks table 
The table (table.1) summarizes the obtained features blocks (B1 to B6) by combining the exposed features modules (b1 to b4).

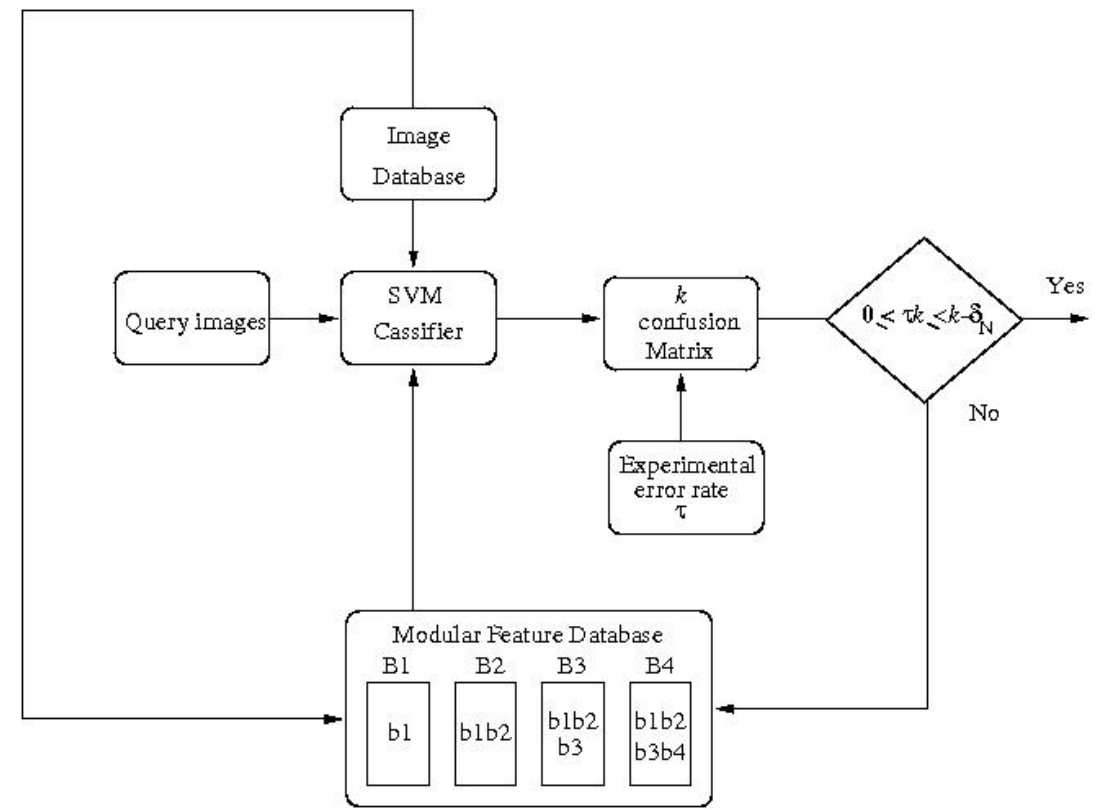

Fig. 9. Modular Statistical optimization architecture.

We can remark in figure 10, for the request (query) image number 4 that the classification rate error is very important for bloc B1. However, the rate error decrease progressively when the others features bloc are used. The presented modular architecture presents some disadvantages, the query images must be included in the database, the experimental rate error is used as prior information. To solve this problem, we propose in the next section the classification procedure based on hierarchical method using the best feature type selection.

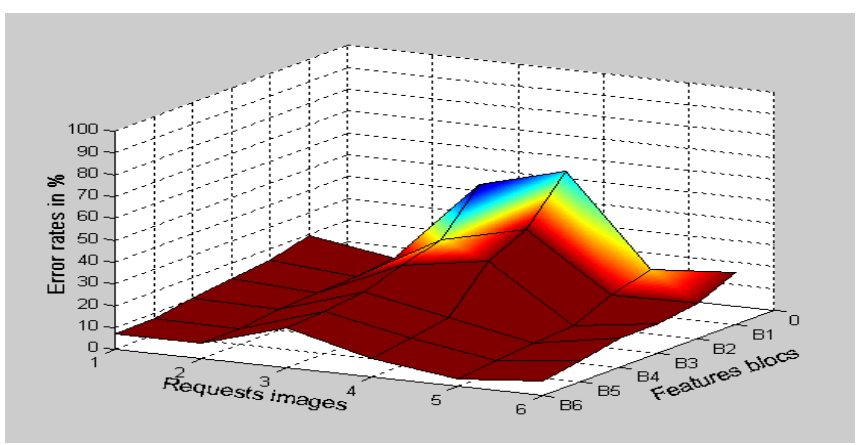

Fig. 10. Average of the classification rate error obtained for different feature blocs. 


\subsection{Best feature type selection method}

The hierarchical feature model is proposed to replace the classical employment of aggregated features (Djouak et al., 2005a), (Djouak et al., 2005b). This method is able to select features and organize them automatically through their kinds and the image database contents. In the off-line stage, due to feature extraction step, we obtain from an image database correspond feature dataset. Then, we start, first of all by the training step, using, at every turn, one group of same feature kind separately, and based on the training rate criterion computed through used classifier, we select hierarchically the best same feature kind. In the on-line stage, we classify each image from the test image database, using separately the different same feature kinds. So, for each image, we will have different clusters as a retrieval result. Then To decide between these various outputs, we treat each two same feature kind outputs together, according to the hierarchical feature selection model, as described in figure 11. We start process within the two latest same feature kind outputs, until reaching the best one. Each time, according to the examined two group of same feature kind outputs, a comparison block, will decide the use of Nearest Cluster Center (NCC) block or not. The NCC block ensure the computation of Euclidian distance between the candidate image and the two cluster centers (clusters used are the two group of same feature kind outputs).

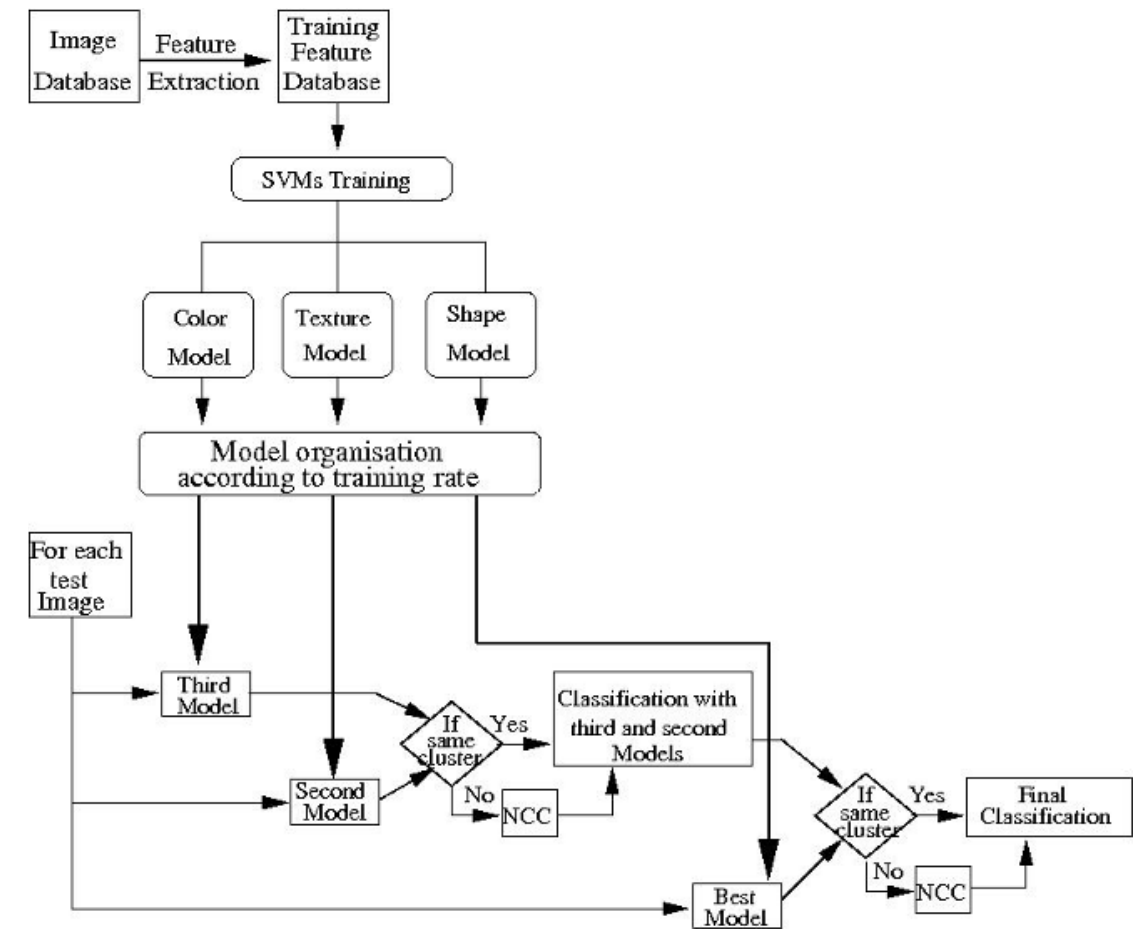

Fig. 11. Hierarchical best features type selection and organization architecture using different SVM models. 
A comparison between classical mixed features and the proposed hierarchical feature model is applied. Hierarchical feature model (figure 11) outperforms the use of aggregated features (several feature kind combination) simply by mixing them all together (color + texture + shape). We present, in Figure 12 and Figure 13, the first 15 images retrieved for a query image, using respectively aggregated features and hierarchical features. In these two figures, the first image is the request image. We observe, obviously, that the retrieval accuracy of hierarchical feature model is more efficient than that of aggregated feature use. However, we demonstrate in this section that the feature aggregation is not enough efficient, if we just mix various feature kind. Indeed, each descriptor kind range is different than those of the other descriptor kinds.

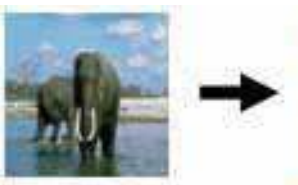

Fig. 12. Retrieval examples, using classical aggregated features.

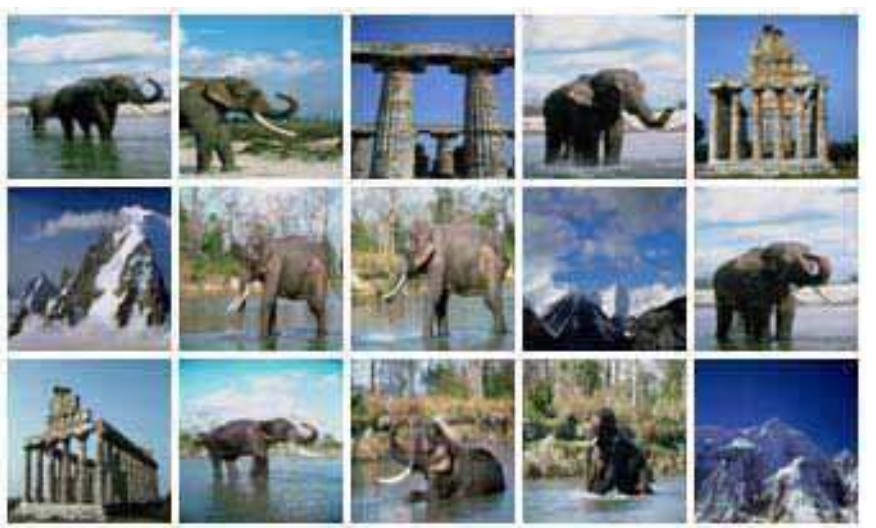

So, each feature vector extracts signature which is not uniform with the other feature signature extracted from images.
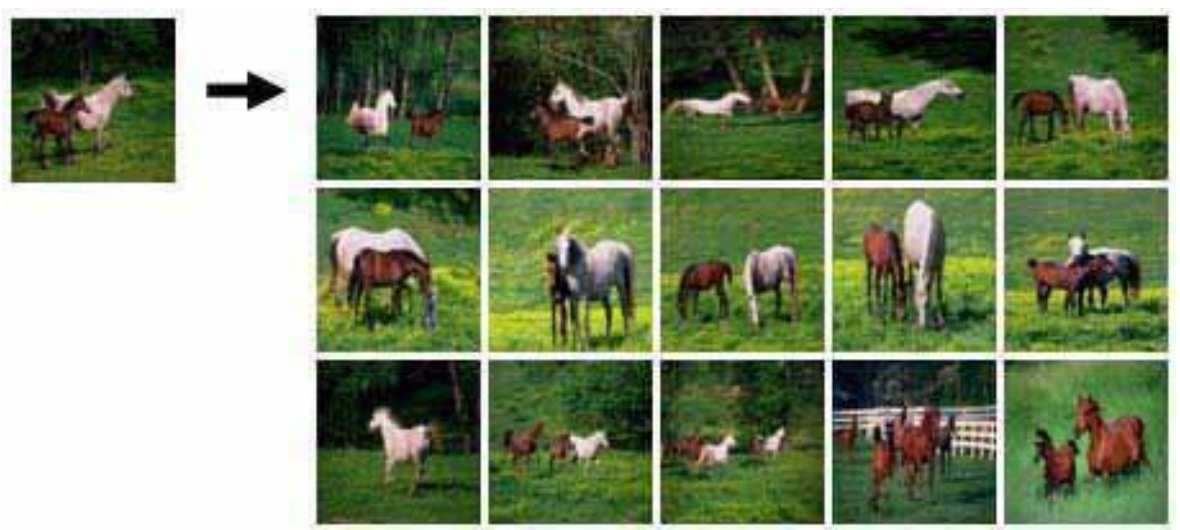

Fig. 13. Retrieval examples, using hierarchical feature model. 
Consequently, we prove that using proposed hierarchical feature model is more efficient than using aggregated features in an heterogeneous image retrieval system.

Figure 14 proves that using the hierarchical feature model is more efficient than using aggregated features in an image retrieval system. Indeed, we obtain with hierarchical features model $0,815 \%$ representing the good classification results and 0,68 \% with aggregated features method.

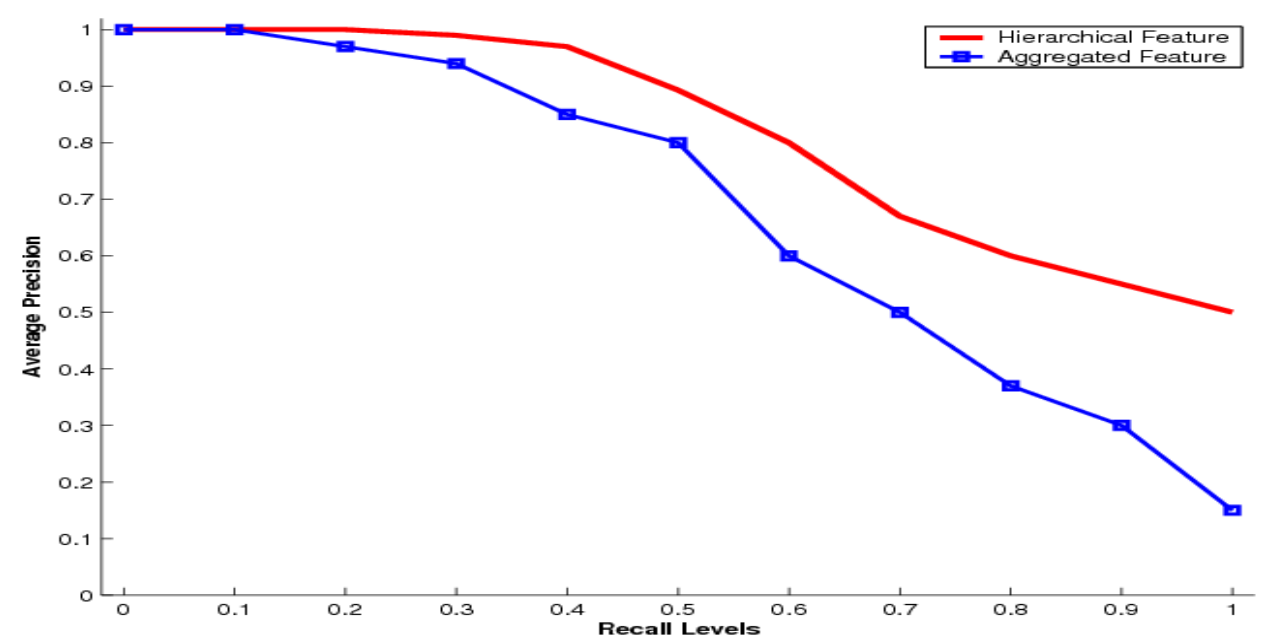

Fig. 14. Precision-recall graph comparing hierarchical features and Aggregated Features.

\section{Conclusion}

In this chapter, we have presented the different stages of image recognition and retrieval system dedicated to different applications based computer vision domain. The image description and classification constitute the two important steps of an image recognition system in large heterogeneous databases. We have detailed the principles of the features extraction, image description contained in large database and the importance of robustness.

After presenting the features extraction and some improvement we have detailed the importance of the classification task and presented the supervised SVM classifier.

To ensure a good feature selection during image retrieval, we have presented and discussed the effectiveness of the different feature kind and aggregation. We have detailed the need of the optimization methods in CBIR systems and we have proposed two architectures, the modular statistical optimization and the hierarchical features model. The satisfactory obtained results show the importance of optimization and the features selection in this domain.

Searching CBIR systems remain a challenges problem. Indeed, the different domains has been unable to take advantage of image retrieval and recognition methods and systems in spite of their acknowledged importance in the face of growing use of image databases in mobile robotics, research, and education. The challenging type of images to be treated and the lacking of suitable systems have hindered their acceptance. While it is difficult to develop a single comprehensive system, it may be possible to take advantage of the growing 
research interest and several successful systems with developed techniques for image recognition in large databases.

\section{References}

Antania, S., Kasturi, R. \& Jain, R. (2002). A survey on the use of pattern recognition methods for abstraction, indexing and retrieval of images and video, Pattern recognition, 35(4), pages: 945-965.

Bi J., Bennett K., Embrechts M., Breneman C., \& Song M., (2003), Dimensionality reduction via sparse support vector machines, J. Machine Learning Research (JMLR), 3, 12291243.

Bimbo A. D., Visual Information Retrieval, (2001), Morgan Kaufmann Publishers, San Francisco, USA.

Blum A.L. \& Langley P., (1997), Selection of Relevant Features and Examples in Machine Learning, Artificial Intelligence, 97(1- 2), 245-271.

Carson, C., Belongie, Se., Greenpan, H. \& Jitendra, M. (2002). Blobworld: Image segmentation using Expectation-Maximization and its Application to Image Querying, IEEE Transaction on Pattern Analysis and Machine Intelligence, Vol. 24, NO. 8.

Chen Y. \& Wang J.Z., (2004), Image Categorization by Learning and Reasoning with Regions, J. Machine Learning Research, vol. 5, pp. 913-939.

Chen Y., Bi J. \& Wang J.Z., (2006), MILES: Multiple-Instance Learning via Embedded Instance Selection, IEEE Transactions on Pattern Analysis and Machine Intelligence, vol. 28, no. 12, pp. 1931-1947.

Cristianini N. \& Taylor J. S., (2000), An Introduction to Support Vector Machines and Other Kernel-Based Learning Methods, Cambridge University Press.

Daphne K. and Mehran S., (1996), Toward optimal feature selection, In International Conference on Machine Learning, 284-292.

Delingette H. \& Montagnat J., (2001), Shape and topology constraints on parametric active contours, Computer Vision and Image Understanding, vol. 83, no. 2, 140-171.

Djouak, A., Djemal, K. \& Maaref, H. (2007). Image Recognition based on features extraction and RBF classifier, Journal Transactions on Signals, Systems and Devices, Issues on Comminucation and Signal Processing, Shaker Verlag, Vol. 2, N. 3, pp: 235-253.

Djouak, A., Djemal, K. \& Maaref, H., (2005a), Image retrieval based on features extraction and RBF classifiers, IEEE International Conference on Signals Systems and Devices, SSD 05 , Sousse. Tunisia.

Djouak, A., Djemal, K. \& Maaref, H.k, (2006). Modular statistical optimization and VQ method for images recognition, International Conference on Artificial Neural Networks and Intelligent Information Processing, pp: 13-24, ISBN: 978-972-8865-689, Setúbal, Portugal, August.

Djouak, A., Djemal K. \& Maaref, H., (2005b), Features extraction and supervised classification intended to image retrieval. In IMACS World Congress: Scientific Computation, Applied Mathematics and Simulation, Paris, France.

Egmont-Petersen, M., de Ridder \& Handels, D. H. (2002). Image processing with neural networks-a review. Pattern Recognition, 35(10):2279-2301. 
Faloutsos C., Equitz W., Flickner M., Niblack W., Petkovic D. \& Barber R., (1994), Efficient and Effective Querying by Image Content, Journal of Intelligent Information Systems, vol. 3, No 3/4, 231- 262.

Guyon I.,Weston J., Barnhill S., \& Vapnik V., (2002), Gene selection for cancer classifcation using support vector machines, Machine Learning 46, 389-422.

Guyon I., \& Elisseeff A., (2003), An introduction to feature and variable selection, Journal of Machine Learning Research, vol. 3, 1157-1182.

Hafner J., Sawhney H.S., Equitz W., Flickner M., \& Niblack W., (1995), Efficient color histogram indexing for quadratic form distance function, IEEE Trans. Pattern Anal. Mach. Intell., 17, 7, 729-736.

Haralick R.M.S.K., \& Dinstein I., (1973), Textural Features for Image Classification, IEEE Transactions on Systems, Man and Cybernetics, 3(6), 610-621.

Hu M.K., (1962), Visual pattern recognition by moment invariants, IEEE Transactions information Theory, 8, 179-187.

Huang J., Kumar S.R., Mitra M., \& Zhu W.J., Spatial color indexing and applications, (1999), Intl. Conf. on Computer Vision, 35, 3, 245-268.

Huang, J., Kumar, S. R., Mitra, M., Zhu, W.-J. \& Zabih, R., (1997). Image indexing using color correlograms. In Proc. IEEE Comp. Soc. Conf. Comp. Vis. and Patt. Rec., pages 762-768.

Jacobs, C., Finkelstein, A. \& Salesin, D. (1995). Fast multiresolution image querying. In Proc. SIGGRAPH.

Julezs. B., (1975), Experiments in the visual perception of texture. Scientific American, 232(4):2-11.

John G.H., Kohavi R., \& Pfleger K., Irrelevant features and the subset selection problem, 1994, In International Conference on Machine Learning, pages 121-129. Journal version in AIJ, available at http :/ / citeseer. nj.nec.com/13663.html.

Kachouri R., Djemal K., Maaref H., Sellami Masmoudi D., \& Derbel N., (2008b), Heterogeneous Image Retrieval System Based On Features Extraction and SVM Classifier, International Conference on Informatics in Control, Automation and Robotics, ICINCO 08, Funchal, Madeira, Portugal.

Kachouri R., Djemal K., Sellami Masmoudi D., \& Derbel N., (2008c), Content Based Image Recognition based on QUIP-tree Model, IEEE International Conference on Signals Systems and Devices, SSD 08, Amman, Jordan.

Kachouri R., Djemal K., Maaref H., Masmoudi, D.S., Derbel N., (2008), Content description and classification for Image recognition system, 3rd IEEE International Conference on Information and Communication Technologies: From Theory to Applications, ICTTA08 14.

Kadyrov, A. \& Petrou, M. (2001). The Trace transform and its applications. IEEE Transactions on Pattern Analysis and Machine Intelligence, PAMI, Vol:811-828.

Lin, Chun-Yi., Yin, Jun-Xun., Gao, X., Chen, Jian-Y. \& Qin, P. (2006). A Semantic Modeling Approach for Medical Image Semantic Retrieval Using Hybrid Bayesian Networks, Sixth International Conference on Intelligent Systems Design and Applications (ISDA'06), vol. 2, pp.482-487.

Lipson, P., Grimson, E. \& Sinha, P. (1997). Configuration based scene classification and image indexing. In Proc. IEEE Comp. Soc. Conf. Comp. Vis. and Patt. Rec., pages 10071013. 
Manjunath B.S., Ohm J.-R., Vasudevan V.V., \& Yamada A., (2001), Color and texture descriptors, IEEE transaction on circuits and systems for video technology, 11, 703-715.

Meng J.E., Shiqian W., Juwei L., \& Hock L.T., (2002), Face Recognition With Radial Basis Function (RBF) Neural Network, IEEE Transaction on Neural Networks, 13, 3.

Press W.H., Flannery B.P., Teukolsky S.A., \& Vetterling W.T., (1987), Numerical Recipes, The Art of Scientific Computing, Cambrigde, U.K.: Cambridge Univ.

Rezai-Rad G., \& Aghababaie M., (2006), Comparison of SUSAN and Sobel Edge Detection in MRI Images for Feature Extraction, Information and Communication Technologies, ICTTA 06. 1, 1103-1107.

Ramesh J. R., Murthy S.N.J., Chen P.L.-J., \& Chatterjee S., (1995), Similarity Measures for Image Databases, Proceedings of IEEE International Joint Conference of the Fourth IEEE International Conference on Fuzzy Systems and The Second International Fuzzy Engineering Symposium, 3, 1247-1254.

Sastry, Ch. S., Pujari, Arun K., Deekshatulu, B. L. \& Bhagvati, C. (2004). A wavelet based multiresolution algorithm for rotation invariant feature extraction, Pattern Recognition Letters, 25:1845-1855.

Sclaroff, S. \& Pentland, A. (1995). Modal Matching for Correspondence and Recognition, IEEE Trans. Pattern Analysis and Machine Intelligence, 17(6):545-561.

Serrano N., Savakisb A.E., \& Luoc J., (2004), Improved scene classification using efficient low-level features and semantic cues, Pattern Recognition, 37 , 1773-1784.

Smith, J. R. \& Chang, S.-F. (1996). Tools and techniques for color image retrieval. In SPIE Proc. Storage and Retrieval for Image and Video Databases, volume 2670, pages 426-437.

Stricker, M. \& Dimai, A. (1997). Spectral covariance and fuzzy regions for image indexing. Machine Vision and Applications, 10(2):66-73.

Stricker, M., \& Swain, M. (1994). Capacity and the sensitivity of color histogram indexing, Technical Report, 94-05, University of Chicago.

Swain, M. \& Ballard, D. (1991). Color indexing, International Journal of Computer Vision, 7(1), pages:11-32.

Takashi, I. \& Masafumi, H. (2000). Content-based image retrieval system using neural networks. International Journal of Neural Systems, 10(5):417-424.

Vapnik, V., (1998). Statistical learning theory, Wiley-Interscience.

Wu, J., (2003), Rotation Invariant Classification of 3D Surface Texture Using Photometric Stereo. PhD Thesis, Heriot-Watt University.

Yu L. \& Liu H., (2004), Efficient Feature Selection via Analysis of Relevance and Redundancy, J. Machine Learning Research, vol. 5, pp. 1205-1224.

Zhu J., \& Hastie T., (2003), Classication of gene microarrays by penalized logistic regression, Biostatistics. 


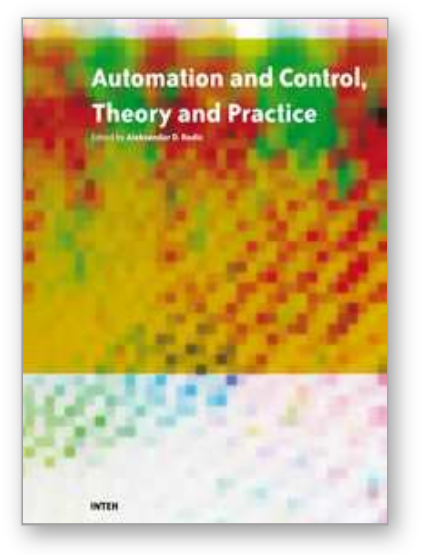

\author{
Automation Control - Theory and Practice \\ Edited by A D Rodi
}

ISBN 978-953-307-039-1

Hard cover, 350 pages

Publisher InTech

Published online 01, December, 2009

Published in print edition December, 2009

The present edited book is a collection of 18 chapters written by internationally recognized experts and wellknown professionals of the field. Chapters contribute to diverse facets of automation and control. The volume is organized in four parts according to the main subjects, regarding the recent advances in this field of engineering. The first thematic part of the book is devoted to automation. This includes solving of assembly line balancing problem and design of software architecture for cognitive assembling in production systems. The second part of the book concerns different aspects of modelling and control. This includes a study on modelling pollutant emission of diesel engine, development of a PLC program obtained from DEVS model, control networks for digital home, automatic control of temperature and flow in heat exchanger, and non-linear analysis and design of phase locked loops. The third part addresses issues of parameter estimation and filter design, including methods for parameters estimation, control and design of the wave digital filters. The fourth part presents new results in the intelligent control. This includes building a neural PDF strategy for hydroelectric satation simulator, intelligent network system for process control, neural generalized predictive control for industrial processes, intelligent system for forecasting, diagnosis and decision making based on neural networks and self-organizing maps, development of a smart semantic middleware for the Internet , development of appropriate Al methods in fault-tollerant control, building expert system in rotary railcar dumpers, expert system for plant asset management, and building of a image retrieval system in heterogeneous database. The content of this thematic book admirably reflects the complementary aspects of theory and practice which have taken place in the last years. Certainly, the content of this book will serve as a valuable overview of theoretical and practical methods in control and automation to those who deal with engineering and research in this field of activities.

\title{
How to reference
}

In order to correctly reference this scholarly work, feel free to copy and paste the following:

Khalifa Djemal, Hichem Maaref and Rostom Kachouri (2009). Image Retrieval System in Heterogeneous Database, Automation Control - Theory and Practice, A D Rodi (Ed.), ISBN: 978-953-307-039-1, InTech, Available from: http://www.intechopen.com/books/automation-control-theory-and-practice/image-retrievalsystem-in-heterogeneous-database

\section{INTECH}

open science | open minds

\section{InTech Europe}

University Campus STeP Ri
InTech China

Unit 405, Office Block, Hotel Equatorial Shanghai 
Slavka Krautzeka 83/A

51000 Rijeka, Croatia

Phone: +385 (51) 770447

Fax: +385 (51) 686166

www.intechopen.com
No.65, Yan An Road (West), Shanghai, 200040, China 中国上海市延安西路65号上海国际贵都大饭店办公楼 405 单元

Phone: +86-21-62489820

Fax: +86-21-62489821 
(C) 2009 The Author(s). Licensee IntechOpen. This chapter is distributed under the terms of the Creative Commons Attribution-NonCommercial-ShareAlike-3.0 License, which permits use, distribution and reproduction for non-commercial purposes, provided the original is properly cited and derivative works building on this content are distributed under the same license. 